\title{
KO-theory of Hermitian symmetric spaces
}

\author{
Akira KonO and Shin-ichiro HARA \\ (Received January 21, 1991)
}

Dedicated to Professor Haruo Suzuki on his 60th birthday

\section{§1. Introduction}

Our purpose of this paper is the determination of $\mathrm{KO}$-theory of the compact irreducible Hermitian symmetric spaces. The spaces are classified by E. Cartan as follows :

$\begin{array}{llll}\text { AIII } M_{m, n}= & U(m+n) /(U(m) \times U(n)) & & \\ B D I \quad Q_{n}= & S O(n+2) /(S O(n) \times S O(2)) & & (n \geq 3) \\ C I & & S p(n) / U(n) & (n \geq 3) \\ \text { DIII } & S O(2 n) / U(n) & (n \geq 4) \\ \text { EIII } \quad=E_{6} /\left(\operatorname{Spin}(10) \cdot T^{1}\right) & & \left(\operatorname{Spin}(10) \cap T^{1} \cong \boldsymbol{Z}_{4}\right) \\ \text { EVII } \quad=E_{7} /\left(E_{6} \cdot T_{1}\right) & & \left(E_{6} \cap T^{1} \cong Z_{3}\right) .\end{array}$

Bott showed their cohomology rings have no torsion and no odd dimensional part. The integral cohomology rings are determined by [2], [9] and [10], while the actions of the squaring operations on them are determined in [5]. In [6], we compute the $K O$-theory of $M_{m, n}$. Here we show :

THEOREM 1. Let $X$ be a compact irreducible Hermitian symmetric space, then its Atiyah-Hirzebruch spectral sequence for $K O^{*}(X)$ :

$$
E_{r}^{*, *}(X) \Rightarrow K O^{*}(X)
$$

has nontrivial differential $d_{r}$ only for $r=2$.

Let $H^{*}(X)$ be the modulo 2 cohomology ring of $X$. When the odd dimensional parts of $H^{*}(X)$ are trivial, $S q^{2} S q^{2}\left(=S q^{3} S q^{1}\right)$ vanishes on $H^{*}(X)$, and $\left(H^{*}(X), S q^{2}\right)$ is a differential module. For the proof of Theorem 1 we compute the (co)homology group $H\left(H^{*}(X) ; S q^{2}\right)$, which is isomorphic to $E_{3}^{*,-1}(X)$, and show the differentials $d_{r}(r \geq 3)$ are trivial for each $X$.

By Theorem 1, $K O^{*}(X)$ is obtained from $E_{3}^{*, *}(X)$. Consequently the groups $H^{*}(X)$ and $H\left(H^{*}(X), S q^{2}\right)$ determine $K O^{*}(X)$ in the following corollary. 
COROLlaRY 2. The $K O^{i}(X)$ is given by the following table:

\begin{tabular}{|c|c|}
\hline$i$ & $K O^{i}$ \\
\hline 0 & $t_{0} \boldsymbol{Z} \oplus s_{1} \boldsymbol{Z}_{2}$ \\
-1 & $s_{0} \boldsymbol{Z}_{2}$ \\
-2 & $t_{1} \boldsymbol{Z} \oplus s_{0} \boldsymbol{Z}_{2}$ \\
-3 & $s_{3} \boldsymbol{Z}_{2}$ \\
-4 & $t_{0} \boldsymbol{Z} \oplus s_{3} \boldsymbol{Z}_{2}$ \\
-5 & $s_{2} \boldsymbol{Z}_{2}$ \\
-6 & $t_{1} \boldsymbol{Z} \oplus s_{2} \boldsymbol{Z}_{2}$ \\
-7 & $s_{1} \boldsymbol{Z}_{2}$ \\
\hline
\end{tabular}

where

$$
\begin{aligned}
t_{\delta} & =\operatorname{dim}_{z_{2}} \bigoplus_{i \equiv 2 \delta(\bmod 4)} H^{i}(X) \\
s_{\varepsilon} & =\operatorname{dim}_{Z_{2}} \oplus_{i \equiv 2 \varepsilon(\bmod 8)} H^{i}\left(H^{*}(X) ; S q^{2}\right) .
\end{aligned}
$$

We discribe $H^{*}(X), H\left(H^{*}(X): S q^{2}\right)$, $t_{\delta}$ and $s_{\varepsilon}$ for each $X$ later.

\section{$\S 1$. Preliminaries}

In this section we recall the result of our previous papers and prepare a lemma for the proof of the theorem.

LEMMA 1.1. Let $X$ be a CW complex of finite type, such that its cohomology has no torsion and is concentrated in even dimensions, and let $E_{r}^{*, *}$ be the Atiyah-Hirzebruch spectral sequence for KO-theory.

(1) We have an isomorphism:

$$
\iota: E_{3}^{*, q} \stackrel{\cong}{\longrightarrow} H\left(H^{*}(X) ; S q^{2}\right), q \equiv-1 \quad(\bmod 8) .
$$

(2) Suppose there is a nontrivial differential $d_{r}: E_{r}^{*, *} \longrightarrow E_{r}^{*+r, *+1-r}$ $(r \geq 3)$. For the smallest $r\left(E_{r}^{*, *}=E_{3}^{*, *}\right)$, the next conditions are satisfied.

(i ) $1-r \equiv-1(\bmod 8)$.

(ii) If $p$ is the smallest integer such that $d_{r}\left(E_{r}^{p, *}\right) \neq\{0\}$, there is an element $x \in E_{r}^{p, 0}$ such that $\eta \cdot x \neq 0$ and $\eta \cdot d_{r} x \neq 0$, where $\eta$ is the generator of the coefficient group $K O^{-1} \cong Z_{2}$.

(iii) $\iota(\eta \cdot x)$ is indecomposable as a element of $H\left(H^{*}(X) ; S q^{2}\right)$.

(3) Moreover if $X$ is a Hopf space, $H\left(H^{*}(X)\right.$; $\left.S q^{2}\right)$ is a Hopf algebra and, in (2), c $\left(d_{r} x\right)$ is a primitive element.

Proof: (1). This is given in [3]. 
(2). (i) and (ii) are demonstrated in [6]. (iii). Suppose $\eta \cdot x$ is a decomposable element. It is written as $\sum \eta \cdot x^{\prime} x^{\prime \prime}$ with $x^{\prime}, x^{\prime \prime} \in E_{r}^{*, 0}$ and deg $x^{\prime}$, deg $x^{\prime \prime}<\operatorname{deg} x$. By the assumption on $p, d_{r} x^{\prime}=d_{r} x^{\prime \prime}=0$. We obtain $d_{r} x=\Sigma \eta \cdot d_{r}\left(x^{\prime}\right) x^{\prime \prime}+\Sigma \eta \cdot x^{\prime} d_{r}\left(x^{\prime \prime}\right)=0$. This is a contradicitn. Thus $\eta \cdot x \in$ $E_{r}^{*,-1}$ is an indecomposable element of the algebra $E_{r}^{*, *}$. Let $A$ be $H\left(H^{*}(X) ; S q^{2}\right)$. Because $H^{*}(\mathrm{X})$ has trivial odd dimensional parts, $S q^{2}$ acts as a derivation on it and its homology group $A$ is an algebra. The product of $A$ is compatible with that of $E_{r}^{*, *}$, that is, the next diagram is commutative :

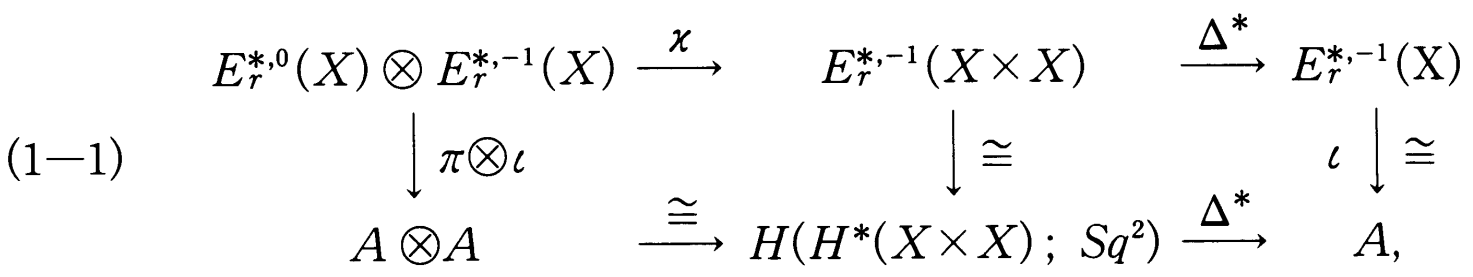

where $x$ is the external product, $\Delta$ is the diagonal map, and $\pi$ is the natural projection:

$$
E_{r}^{*, 0}(X)=E_{3}^{*, 0}(X) \cong \operatorname{Ker}\left[S q^{2} \pi_{2}: H^{*}(X ; \boldsymbol{Z}) \rightarrow H^{*+2}(X)\right] \rightarrow H\left(H^{*}(X) ; S q^{2}\right) .
$$

$\left(\pi_{2}\right.$ is the modulo 2 reduction $H^{*}(X ; \boldsymbol{Z}) \rightarrow H^{*}(X)$.) The map $\pi \otimes_{\iota}$ is epimorphic. This proves that: if $\eta \cdot x$ is indecomposable then $\iota(\eta \cdot x)$ is also indecomposable.

(3). Since $H^{*}(X)$ is a Hopf algebra and $S q^{2}$ is a derivation and commutes with the coproduct, $A$ has a Hopf algebra structure. Let $\psi$ : $E_{r}^{* * *}(X) \rightarrow E_{r}^{*, *}(X \times X)$ be the map induced by the multiplication of $X$. Consider the commutative diagram :

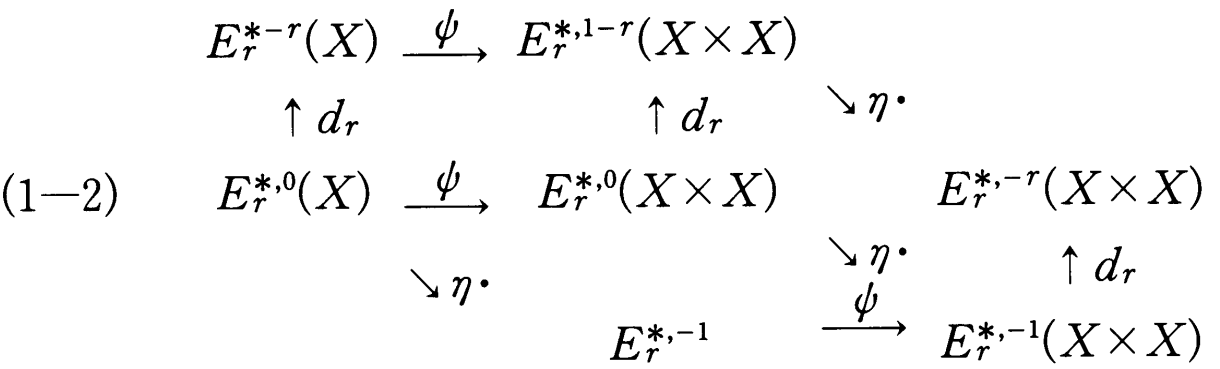

As the external product map $x$ is an epimorphism by the diagram (1 $-1), \psi(\eta \cdot x) \in E_{r}^{*,-1}(X \times X)$ can be expressed as :

$$
\psi(\eta \cdot x)=\eta \cdot x \otimes 1+1 \otimes \eta \cdot x+\sum x^{\prime} \otimes x^{\prime \prime} \quad\left(x^{\prime} \in E_{r}^{*,-1}(X)\right)
$$

( $x$ is omitted.) By assumption on $p, d_{r} x^{\prime}=d_{r} x^{\prime \prime}=0$, thus we have $d_{r} \psi(\eta \cdot x)$ $=d_{r}(\eta \cdot x) \otimes 1+1 \otimes d_{r}(\eta \cdot x)$ and $\eta \cdot d_{r} \psi(x)=\eta \cdot\left(d_{r} x \otimes 1+1 \otimes d_{r} x\right)$. Since the 
multiplication by $\eta: E_{r}^{*, 1-r}(X \times X) \rightarrow E_{r}^{*,-r}(X \times X)$ is a monomorphism, we obtain $d_{r} \psi(x)=d_{r} x \otimes 1+1 \otimes d_{r} x=\psi\left(d_{r} x\right)$. It follows that $\iota\left(d_{r} x\right)$ is primitive as an element of $A$.

\section{$\S 2$. Type CI, DIII and BDI}

In this section we show the collapsing of the Atiyah-Hirzebruch spectral sequence for the spaces of the classical types. (For the case of $M_{m, n}$, it is done in [6].)

First we consider the space of type CI, $S p(n) / U(n)$. Recall that the modulo 2 cohomology of $S p(n) / U(n)$ is :

$$
H^{*}(S p(n) / U(n)) \cong \wedge\left(c_{1}, c_{2}, \cdots, c_{n}\right),
$$

where $c_{i}$ is the $\mathrm{i}$-th Chern class.

Define the differential submodules $M_{j}$ of $H^{*}(S p(n) / U(n))$ by

$$
M_{j}=\wedge\left(c_{2 j}, c^{\prime}{ }_{2 j+1}\right),
$$

where $c^{\prime}{ }_{2 j+1}=S q^{2} c_{2 j}=c_{2 j+1}+c_{2 j} c_{1}, j \geq 1$. Let $m=[n / 2]$, then we have

$$
H^{*}(S p(n) / U(n)) \cong \begin{cases}\wedge\left(c_{1}, c_{2 m}\right) \otimes M_{1} \otimes M_{2} \otimes \cdots \otimes M_{m-1}, & \text { if } n=2 m, \\ \bigwedge\left(c_{1}\right) \otimes M_{1} \otimes M_{2} \otimes \cdots \otimes M_{m}, & \text { if } n=2 m+1 .\end{cases}
$$

As $H\left(M_{j} ; S q^{2}\right) \cong \bigwedge\left(\left[c_{2 i} c^{\prime}{ }_{2 i+1}\right]\right)$, we have :

$H\left(H^{*}(S p(n) / U(n)) ; S q^{2}\right) \cong \begin{cases}\bigwedge\left(\left[c_{1}\right],\left[c_{2} c_{3}^{\prime}\right], \cdots,\left[c_{2 m-2} c_{2 m-1}^{\prime}\right]\right), & \text { if } n=2 m, \\ \bigwedge\left(\left[c_{1}\right],\left[c_{2} c_{3}^{\prime}\right], \cdots,\left[c_{2 m} c_{2 m+1}^{\prime}\right]\right), & \text { if } n=2 m+1 .\end{cases}$

It is easy to see the case for $n=\infty$

$$
H\left(H^{*}(S p / U) ; S p^{2}\right) \cong \bigwedge\left(\left[c_{1}\right],\left[c_{2} c_{3}^{\prime}\right], \cdots,\left[c_{2 j} c^{\prime}{ }_{2 j+1}\right], \cdots\right) .
$$

Since $S p / U$ has a homotopy commutative Hopf space structure (in fact, it is an infinite loop space) and all generators have the form of $\left[c_{2 j} c_{2 j+1}^{\prime}\right]$, whose degrees are in $8 j+2$. By Lemma 1.1, if the Atiyah-Hirzebruch spectral sequence has nontrivial differentials $d_{r}$, for the smallest $r$, there must be elements of $H\left(H^{*}(S p / U) ; S q^{2}\right), x, y$, corresponding to the source and the target of $d_{r}$ respectively, such that $\operatorname{deg} y-\operatorname{deg} x=r \equiv 2(\bmod 8)$ and $x$ is a generator and $y$ is a primitive element. But, as the all primitive elements are in the dimensions of the generators [Prop. 4.23, 8], that is impossible. Thus $E_{r}^{*, *}(S p / U)$ collapses for $r \geq 3$. Since the canonical map $E_{r}^{*, *}(S p / U) \rightarrow E_{r}^{*, *}(S p(n) / U(n))$ is an epimorphism. $E_{r}^{*, *}(S p(n) /$ $U(n)), r \geq 3$ collapses. Thus we have : 
THEOREM 2.1. The Atiyah-Hirzebruch spectral sequence for $K O$ theory of $S p(n) / U(n)$ collapses for $r \geq 3$ and

$$
E_{\infty}^{*,-1}(S p(n) / U(n)) \cong \bigwedge\left(x_{2}, x_{10}, \cdots, x_{8 k+2}\right),
$$

where $k=\left[\frac{n-1}{2}\right]$ and deg $x_{i}=i$.

Next we consider the space of type DIII, $S O(2 n) / U(n)$. It is known that $([5])$ :

$(2-3) \quad H^{*}(S O(2 n) / U(n)) \cong \triangle\left(e_{2}, e_{4}, \cdots, e_{2 n-2}\right), \operatorname{deg} e_{i}=i$

where $e_{2 i}{ }^{2}=e_{4 i}$, and $e_{2 j}=0(j \geq n)$, and the action of $S q^{2}$ is given by $S q^{2} e_{2 i}=$ $i \cdot e_{2 i+2 \text {. }}$

Define the differential submodules $M_{i}$ of $H^{*}(S O(2 n) / U(n))$ by

$$
M_{i}=Z_{2}\left\langle 1, e_{4 i-2}, e_{4 i}, e_{8 i-2}^{\prime}\right\rangle,
$$

where $e_{8 i-2}^{\prime}=e_{4 i-2} e_{4 i}+e_{8 i-2}$. Then $H^{*}(S O(2 n) / U(n))$ splits as

$$
H^{*}(S O(2 n) / U(n))= \begin{cases}M_{1} \otimes M_{2} \otimes \cdots \otimes M_{m-1} \otimes \wedge\left(e_{4 m-2}\right) & , \text { if } n=2 m, \\ M_{1} \otimes M_{2} \otimes \cdots \otimes M_{m} & \text {, if } n=2 m+1 .\end{cases}
$$

Using $S q^{2} e_{4 i-2}=e_{4 i}, S q^{2} e_{8 i-2}^{\prime}=0$, we get $H\left(M_{i} ; S q^{2}\right)=Z_{2}\left\langle 1,\left[e_{8 i-2}\right]\right\rangle$. Thus we obtain

$$
H\left(H^{*}(S O(2 n) / U(n)) ; S q^{2}\right)=\left\{\begin{array}{l}
\triangle\left(\left[e_{6}^{\prime}\right],\left[e_{14}^{\prime}\right], \cdots,\left[e_{8 m-10}^{\prime}\right],\left[e_{4 m-2}\right]\right), \text { if } n=2 m, \\
\triangle\left(\left[e_{6}^{\prime}\right],\left[e_{14}^{\prime}\right], \cdots,\left[e_{8 m-2}^{\prime}\right]\right), \quad \text { if } n=2 m+1 .
\end{array}\right.
$$

On the other hand, since $e^{\prime}{ }_{8 i-2}^{2}=S q^{2}\left(e_{8 i-6} e_{8 i}+e_{16 i-6}\right)$, we have $\left[e_{8 i-2}^{\prime}\right]^{2}=0$, and the algebra of $(2-4)$ is an external algebra.

Now we show the Atiyah-Hirzebruch spectral sequence $E_{r}^{*, *}$ collapses for $r>3$. As the similar way for the type CI, consider the case $n=\infty$, then the space $S O / U$ is a Hopf space and $H\left(\left(H^{*}(S O(2 n) / U(n)) ; S q^{2}\right) \cong\right.$ $\wedge_{j \geq 1}\left(\left[e_{8 j-2}^{\prime}\right]\right)$. Since the generators and primitives are concentrated in the degrees $\{8 j-2\}$, by Lemma 1.1, there is no nontrivial differential $d_{r}$ on $E_{r}^{*, *}(S O / U)$ for $(r>3)$. For finite $n$, consider the map

$$
E_{r}^{*, *}(S O / U) \rightarrow E_{r}^{*, *}(S O(2 n) / U(n)),
$$

which is induced by inclusion $S O(2 n) / U(n) \rightarrow S O / U$. The elements $\left[e_{8 j-2}^{\prime}\right]$ in $(2-4)$ is in the image of this map. Therefore the possible nontrivial differential in the minimal degree occurs only on $\left[e_{4 \mathrm{~m}-2}\right]$. We show the next lemma later. 
LEMMA 2.2. $\left[e_{4 m-2}\right] \in E_{3}^{*,-1}(S O(4 m) / U(2 m))$ is a permanent cycle.

This completes the proof of this theorem.

THEOREM 2.3. The Atiyah-Hirzebruch spectral sequence for $K O$ theory of $S O(2 n) / U(n)$ collapses for $r \geq 3$ and

$$
E_{\infty}^{*,-1}(S O(2 n) / U(n)) \cong \begin{cases}\bigwedge\left(x_{6}, x_{14}, \cdots, x_{8 m-10}, y_{4 m-2}\right), & \text { if } n=2 m, \\ \bigwedge\left(x_{6}, x_{14}, \cdots, x_{8 m-2}\right), & \text { if } n=2 m+1\end{cases}
$$

where deg $x_{i}=i$ and deg $y_{i}=i$.

Lastly we consider the space of type BDI :

$$
Q_{n}=S O(n+2) /(S O(n) \times S O(2))
$$

Let $t \in H^{2}(B S O(2) ; Z)$ be the canonical generator and put $t=\iota^{*}(1 \times t) \in$ $H^{*}\left(Q_{n} ; Z\right)$, where $\iota$ comes from the fibration:

$$
Q_{n} \stackrel{\iota}{\longrightarrow} B O(n) \times B O(2) \longrightarrow B O(n+2)
$$

When $n=2 m$, let $\chi \in H^{2 m}(B S O(2 m) ; Z)$ be the Euler class. There is an element $s_{2 m} \in H^{2 m}\left(Q_{2 m} ; Z\right)$ such that $2 s_{2 m}=\iota^{*}\left(\chi \times 1+1 \times t^{n}\right)$. The same symbol $s_{2 m} \in H^{2 m}\left(Q_{2 m}\right)$ denotes its modulo 2 reduction. When $n=2 m-1$, $s_{2 m} \in H^{2 m}\left(Q_{2 m-1}\right)$ denotes its image by the map induced by the inclusion $i$ : $Q_{2 m-1} \rightarrow Q_{2 m}$.

It is known that $([5])$ :

$(2-5) \quad H^{*}\left(Q_{n}\right)= \begin{cases}Z_{2}\left[t, s_{4 m}\right] /\left(t^{2 m+1}, s_{4 m}^{2}-s_{4 m} t^{2 m}\right), & \text { if } n=4 m, \\ Z_{2}\left[t, s_{4 m}\right] /\left(t^{2 m}, s_{4 m}{ }^{2}\right), & \text { if } n=4 m-1, \\ Z_{2}\left[t, s_{4 m-2}\right] /\left(t^{2 m}, s_{4 m-2}{ }^{2}\right), & \text { if } n=4 m-2, \\ Z_{2}\left[t, s_{4 m-2}\right] /\left(t^{2 m-1}, s_{4 m-2}{ }^{2}\right), & \text { if } n=4 m-3 .\end{cases}$

and

$$
S q^{2} s_{2 k}=(k+1) s_{2 k} t .
$$

From this, we can easily compute the $S q^{2}$ cohomology of them.

$(2-6) \quad H\left(H^{*}\left(Q_{n}\right) ; S q^{2}\right)= \begin{cases}\left.\wedge\left[t^{2 m} s_{4 m}\right]\right), & \text { if } n=4 m, \\ \left.\wedge\left[t^{2 m-1}\right]\right), & \text { if } n=4 m-1, \\ \left.\wedge\left[t^{2 m-1}\right],\left[s_{4 m-2}\right]\right), & \text { if } n=4 m-2, \\ \wedge\left(\left[s_{4 m-2}\right]\right), & \text { if } n=4 m-3 .\end{cases}$

In the cases other than $n=4 m-2$, it is trivial that the AtyahHirzegruch spectral sequence $E_{r}^{*, *}(r \geq 3)$ collapses. If $n=4 m-2$, the inclusion map $i: Q_{4 m-2} \rightarrow Q_{4 m-1}$ maps $\left[t^{2 m-1}\right]$ to $\left[t^{2 m-1}\right]$, thus we see $\left[t^{2 m-1}\right]$ 
is a permanent cycle. Therefore it is enough to show that:

LEMMA 2.4. In $H\left(H^{*}\left(Q_{4 m-2}\right) ; S q^{2}\right),\left[s_{4 m-2}\right]$ is a permanent cycle.

We demonstrate it later. Thus we have

THEOREM 2.5. The Atiyah-Hirzebruch spectral sequence for KO theory of $Q_{n}$ collapses for $r \geq 3$ and

$$
E_{\infty}^{*,-1}\left(Q_{n}\right) \cong \begin{cases}\wedge\left(z_{8 m}\right), & \text { if } n=4 m, \\ \wedge\left(x_{4 m-2}\right), & \text { if } n=4 m-1, \\ \wedge\left(x_{4 m-2}, y_{4 m-2}\right), & \text { if } n=4 m-2, \\ \wedge\left(y_{4 m-2}\right), & \text { if } n=4 m-3 .\end{cases}
$$

where deg $x_{i}=\operatorname{deg} y_{i}=\operatorname{deg} z_{i}=i$

Now we prove Lemma 2.2 and Lemma 2.4 simultaneously.

Proof of LEMma 2.2. AND Lemma 2.4: Consider the diagram:

$$
\begin{gathered}
S O(4 m) / U(2 m-1) \times U(1) \stackrel{q}{\longrightarrow} S O(4 m) / U(2 m) \\
p \downarrow \\
Q_{4 m-2}=S O(4 m) / S O(4 m-2) \times S O(2)
\end{gathered}
$$

where $p$ and $q$ are the canonical maps. It is easy to see that $H^{*}(q)$ is an injection and $H^{*}(S O(4 m) / U(2 m-1) \times U(1))=H^{*}(S O(4 m) / U(2 m)) \otimes \boldsymbol{Z}_{2}[t] /$ $\left(t^{2 m}\right)$, deg $t=2$.

Apply $H\left(H^{*}(\quad) ; S q^{2}\right)$ to that diagram:

$$
\begin{gathered}
\wedge\left(\left[e_{6}^{\prime}\right], \cdots,\left[e_{8 m-10}^{\prime}\right],\left[e_{4 m-2}\right]\right) \otimes \wedge\left(\left[t^{2 m-1}\right]\right) \stackrel{q^{*}}{p^{*} \uparrow} \wedge\left(\left[e_{6}^{\prime}\right], \cdots,\left[e_{8 m-10}^{\prime}\right],\left[e_{4 m-2}\right]\right) \\
\wedge\left(\left[t^{2 m-1}\right],\left[s_{4 m-2}\right]\right) .
\end{gathered}
$$

Here $p^{*}$ and $q^{*}$ are monomorphisms, and by [5],

$$
\begin{aligned}
p^{*} S_{4 m-1} & =\sum_{i=0}^{2 m-2} e_{4 m-2-2 i} t^{i} \\
& =e_{4 m-2}+\sum_{i=0}^{m-2} S q^{2}\left(e_{4 m-6-4 i} t^{2 i+1}\right) .
\end{aligned}
$$

Thus we obtain $p^{*}\left[s_{4 m-2}\right]=\left[e_{4 m-2}\right]$, and we can take $t$ so as satinfy $p^{*} t=t$.

Suppose there is a nontrivial differential on $E_{r}^{*, *}\left(Q_{4 m-2}\right)(r \geq 3)$. Then, by the differential, $\left[s_{4 m-2}\right]$ corresponds to $\left[t^{2 m-1}\right]\left[s_{4 m-2}\right]$. Thus $\left[e_{4 m-2}\right]$ corresponds to $\left[t^{2 m-1}\right]\left[e_{4 m-2}\right]$ in $H\left(H^{*}(S O(4 m) / U(2 m-1) \times U(1)) ; S q^{2}\right)$. On the other hand, $\left[e_{4 m-2}\right] \in \operatorname{Im} q^{*}$, but $\left[t^{2 m-1}\right]\left[e_{4 m-2}\right] \notin \operatorname{Im} q^{*}$, this is a contradic- 
tion. Therefore $\left[s_{4 m-2}\right]$ is a permanent cycle.

By the same way, we can see the $\left[e_{4 m-2}\right]$ is a permanent cycle.

\section{$\S 3$. Exceptional types}

The integral cohomology ring of EIII is obtained in [9], and the modulo 2 reduction is :

$$
H^{*}(E I I I)=Z_{2}[t, w] /\left(t^{9}+w^{2} t, w^{3}+w^{2} t^{4}+w t^{8}\right),
$$

where $\operatorname{deg} w=8$ and $\operatorname{deg} t=2$, and by [5], $S q^{2} w=w t+t^{5}$.

Let $w^{\prime}=w+t^{4}$, then

$$
H^{*}(E I I I)=\boldsymbol{Z}_{2}\left[t, w^{\prime}\right] /\left(w^{\prime 2} t, w^{\prime 3}+t^{12}\right), \text { with } S q^{2} w^{\prime}=w^{\prime} t .
$$

Thus we have

(3-2) $\left.\quad H\left(H^{*}(E I I I) ; S q^{2}\right)\right)=\boldsymbol{Z}_{2}\left[\left[w^{\prime 2}\right]\right] /\left(\left[w^{\prime 2}\right]^{3}\right)$.

Its generators exist only in the degree 0 modulo 8. Lemma 1.1 assert that the Atiyah-Hirzebruch spectral sequence collapses. We obtain:

THEOREM 3.1. The Atiyah-Hirzebruch spectral sequence for KO theory of EIII collapses for $r \geq 3$ and

$$
E_{\infty}^{*,-1}(E I I I) \cong Z_{2}\left[x_{16}\right] /\left(x_{16}{ }^{3}\right),
$$

where deg $x_{16}=16$.

Lastly we consider the case EVII. From the result of [10], we have $(3-3) \quad H^{*}(E V I I)=Z_{2}[u, v, w] /\left(u^{14}, v^{2}, w^{2}\right)$,

where $\operatorname{deg} u=2, \operatorname{deg} v=10, \operatorname{deg} w=18$, and the actions of cohomology operations are determined in [5],

$$
\begin{array}{ccc}
S q^{2} v=0, & S q^{4} v=v u^{2}+u^{7}, \quad S q^{2} v=w+v u^{4}+u^{9}, \quad \\
S q^{2} w=u^{10}, \quad S q^{4} w=v u^{6}+u^{11}, \quad S q^{8} w=v u^{8}+u^{13}, \quad S q^{16} w=v u^{12} .
\end{array}
$$

Let $w^{\prime}=w+u^{9}$, then

$$
H^{*}(E V I I)=Z_{2}\left[u, v, w^{\prime}\right] /\left(u^{14}, v^{2}, w^{\prime 2}\right) \text {, with } S q^{2} v=0 \text { and } S q^{2} w^{\prime}=0 .
$$

Thus we have $\left.(3-4) \quad H\left(H^{*}(E V I I) ; S q^{2}\right)\right)=\wedge\left(\left[u^{13}\right],[v],\left[w^{\prime}\right]\right)$.

In this algebra the generators are in degrees 26,10 and 18 . So we cannot apply Lemma 1.1 directly to this case. To use the Hopf algebra structure, consider a generating map 


$$
g: E V I I \rightarrow \Omega E_{7}
$$

which makes EVII a generating variety of $\Omega E_{7}$. In [1], Bott showes:

Proposition 3.2. $\operatorname{Im}\left[g^{*}: H_{*}(E V I I) \rightarrow H_{*}\left(\Omega E_{7}\right)\right]$ generates the Pontrjagin ring.

On the other hand, $\Omega E_{7}$ is a homotopy commutative Hopf space and $H_{*}\left(\Omega E_{7}\right)$ is completely given in [7]:

PROPOSITION 3.3.

(1) $H_{*}\left(\Omega E_{7}\right) \cong \bigwedge\left(x_{2}, x_{4}, x_{8}\right) \otimes \boldsymbol{Z}_{2}\left[x_{10}, x_{14}, x_{16}, x_{18}, x_{22}, x_{26}, x_{34}\right]$.

(2) For the coproduct $\phi$,

$$
\begin{aligned}
\phi x_{4}= & x_{4} \otimes 1+x_{2} \otimes x_{2}+1 \otimes x_{4}, \\
\phi x_{8}= & x_{8} \otimes 1+x_{2} x_{4} \otimes x_{2}+x_{2} \otimes x_{2} x_{4}+1 \otimes x_{8}, \\
\phi x_{16}= & x_{16} \otimes 1+x_{2} x_{4} x_{8} \otimes x_{2}+x_{4} x_{8} \otimes x_{4}+x_{2} x_{8} \otimes x_{2} x_{4}+x_{8} \otimes x_{8} \\
& +x_{2} x_{4} \otimes x_{2} x_{8}+x_{4} \otimes x_{4} x_{8}+x_{2} \otimes x_{2} x_{4} x_{8}+1 \otimes x_{16} .
\end{aligned}
$$

Other generators are primitive.

(3) The dual operations are completely determined by:

$S q_{*}^{2} x_{4}=x_{2}, \quad S q_{*}^{2} x_{8}=x_{2} x_{4}, \quad S q_{*}^{2} x_{16}=x_{14}+x_{2} x_{4} x_{8}, \quad S q_{*}^{2} x_{22}=x_{10}^{2}$,

$S q_{*}^{2} x_{34}=x_{16}^{2}$,

$S q_{*}^{4} x_{8}=x_{4}, \quad S q_{*}^{4} x_{14}=x_{10}, \quad S q_{*}^{4} x_{16}=x_{4} x_{8}, \quad S q_{*}^{4} x_{26}=x_{22}$,

$S q_{*}^{8} x_{16}=x_{8}, \quad S q_{*}^{8} x_{18}=x_{10}, \quad S q_{*}^{8} x_{22}=x_{14}, \quad S q_{*}^{8} x_{26}=x_{18}$,

otherwise, $\quad S q_{*}^{2 i} x_{j}=0$,

We can easily compute its dual Hopf algebra from this. Let $w_{i}$ be the dual element of $x_{i}$ for the monomial basis of $x_{i}$ 's, (exceptionally $w_{32}$ be the dual to $x_{16}^{2}$ ).

PROPOSITION 3.4.

(1) $H^{*} \Omega E_{7} \cong Z_{2}\left[w_{2}\right] /\left(w_{2}^{16}\right) \otimes \Gamma\left(w_{10}, w_{14}, w_{18}, w_{22}, w_{26}, w_{32}, w_{34}\right)$,

where $\Gamma(w)$ denotes the divided power algebra which has addtive basis $\left\{\gamma_{n}(w)\right\}$.

(2) The generators indicated above except $w_{32}$ are primitive.

(3) The cohomology operations are given by:

$$
\begin{array}{llll}
\mathrm{S} q^{2} w_{2}=w_{2}{ }^{2}, & \mathrm{~S} q^{2} w_{14}=w_{2}{ }^{8}, & \mathrm{~S} q^{2} \gamma_{2}\left(w_{10}\right)=w_{22}, & \mathrm{~S} q^{2} w_{32}=w_{34}, \\
\mathrm{~S} q^{4} w_{4}=w_{8}, & \mathrm{~S} q^{4} w_{10}=w_{14}, & \mathrm{~S} q^{4} w_{22}=w_{26}, & \\
\mathrm{~S} q^{8} w_{8}=w_{16}, & \mathrm{~S} q^{8} w_{10}=w_{18}, & \mathrm{~S} q^{8} w_{14}=w_{22}, & \mathrm{~S} q^{8} w_{18}=w_{26} .
\end{array}
$$

If $x$ is the dual element of the generator of $H_{*}\left(\Omega E_{7}\right)$, then $g^{*}(x)$ is the non zero element, because $\operatorname{Im} g_{*}$ generates $H_{*}\left(\Omega E_{7}\right)$. We can determine 
$g_{*}$ as follows.

For dimensional reasons, $g^{*}\left(w_{2}\right)=u$. Since $g^{*}\left(w_{10}\right)^{2}=g^{*}\left(w_{10}{ }^{2}\right)=0$, we have :

$$
g^{*}\left(w_{10}\right)=v .
$$

From this $g^{*}$ is determined, using squaring operations :

$$
\begin{aligned}
& g^{*}\left(w_{14}\right)=g^{*}\left(S q^{4} w_{10}\right)=S q^{4} g^{*}\left(w_{10}\right)=S q^{4} v=v u^{2}+u^{7}, \\
& g^{*}\left(w_{18}\right)=g^{*}\left(S q^{8} w_{10}\right)=S q^{8} g^{*}\left(w_{10}\right)=S q^{8} v=w^{\prime}+v u^{4}, \\
& g^{*}\left(w_{22}\right)=g^{*}\left(S q^{8} w_{14}\right)=S q^{8} g^{*}\left(w_{14}\right)=S q^{8}\left(v u^{2}+u^{7}\right)=w^{\prime} u^{2}, \\
& g^{*}\left(w_{26}\right)=g^{*}\left(S q^{4} w_{22}\right)=S q^{4} g^{*}\left(w_{22}\right)=S q^{4}\left(w^{\prime} u^{2}\right)=w^{\prime} u^{4}+v u^{8}+u^{13} .
\end{aligned}
$$

By the way, from Proposition 3.4, we get the next isomorphism of the Hopf algebra.

$$
\left.H\left(H^{*}\left(\Omega E_{7}\right\} ; S q^{2}\right)\right) \cong \bigwedge\left(\left[w_{10}\right],\left[w_{14}+w_{2}^{7}\right]\right) \otimes \Gamma\left(\left[w_{18}\right],\left[w_{26}\right],\left[\gamma_{2}\left(w_{22}\right)\right],\left[\gamma_{2}\left(w_{34}\right)\right]\right) .
$$

From $(3-5)$ and $(3-6)$, we have the correspondence of elements of $S q^{2}$ cohomology groups.

$$
\begin{aligned}
& (3-8) \quad H\left(g^{*} ; S q^{2}\right)\left(\left[w_{18}\right]\right)=\left[w^{\prime}+v u^{4}\right]=\left[w^{\prime}\right], \\
& H\left(g^{*} ; S q^{2}\right)\left(\left[w_{26}\right]\right)=\left[w^{\prime} u^{4}+v u^{8}+u^{13}\right]=\left[u^{13}\right] .
\end{aligned}
$$

Suppose that there is a nontrivial differential of $E_{r}^{*, *}(E V I I)$. By Lemma 1.1 and $(3-4)$, it is given by:

$$
d_{r} \alpha=\beta \text {, with } \operatorname{deg} \alpha=10 \text { or } 18 \text { or } 26 \text {, and } \operatorname{deg} \beta=28 \text { or } 36 \text { or } 44 .
$$

Because $H\left(g^{*} ; S q^{2}\right)$ is epimorphic by $(3-8)$, the differential must occur in the same dimensions of $E_{r}^{*, *}\left(\Omega E_{7}\right)$. Again by Lemma 1.1, the target must be primitive. Thus by $(3-7)$, we conclude that:

$$
\left[\gamma_{2}\left(w_{22}\right)\right] \text { is hit by }\left[w_{10}\right] \text { or }\left[w_{18}\right] \text { or }\left[w_{26}\right] \text {. }
$$

To show that this is impossible, it is enough to prove that $\left[x_{22}{ }^{2}\right]$ is a permanent cycle of the dual Atiyah-Hizebruch spectral sequence $E_{*, *}^{r}\left(\Omega E_{7}\right)$ for $K O_{*}\left(\Omega E_{7}\right)$. Here, we quote the result on $H_{*}\left(\Omega F_{4}\right)$ from [7] again.

PROPOSITION 3.5. $H_{*}\left(\Omega F_{4}\right) \cong \bigwedge\left(x_{2}\right) \otimes Z_{2}\left[x_{4}, x_{10}, x_{14}, x_{22}\right]$.

$$
S q_{*}^{2} x_{4}=x_{2}, S q_{*}^{2} x_{10}=x_{4}^{2}, S q_{*}^{2} x_{22}=x_{10}^{2} .
$$

Thus we have $(3-9) \quad E_{*, *}^{3}\left(\Omega F_{4}\right) \cong H\left(H_{*}\left(\Omega F_{4}\right) ; S q_{*}^{2}\right) \cong Z_{2}\left[\left[x_{14}\right],\left[x_{22}{ }^{2}\right]\right]$. 
As we discussed in Lemma 1.1, this spectral sequence $E_{*, *}^{r}\left(\Omega F_{4}\right)$ collapse for $r \geq 3$, by dimensional reason. So $\left[x_{22}{ }^{2}\right]$ is a permanent cycle. By the canonical inclusion $F_{4} \stackrel{i}{\longrightarrow} E_{7}, x_{22}$ maps to $x_{22}$. Hence $\left[x_{22}{ }^{2}\right]$ is a permanent cycle in $E_{*, *}^{r}\left(\Omega E_{7}\right)$. This completes the proof of the next theorem.

THEOREM 3.6. The Atiyah-Hirzebruch spectral sequence for $K O$ theory of EVII collapses for $r \geq 3$ and

$$
E_{\infty}^{*,-1}(E V I I) \cong \bigwedge\left(x_{10}, x_{18}, x_{26}\right),
$$

where deg $x_{i}=i$.

\section{$\S 4$. Proof of Corollary 2 and lists}

Suppose $X$ is a finite complex such that $H^{*}(X ; \boldsymbol{Z})$ has no torsion and no odd dimensional part. By the similar arguments of Lemma 2.1 and 2 . 2 of [4] we have

$$
\begin{aligned}
& K O^{2 i+1}(X) \cong s \boldsymbol{Z}_{2}, \\
& K O^{2 i}(X) \cong r \boldsymbol{Z} \otimes s \boldsymbol{Z}_{2}
\end{aligned}
$$

for some $r$ and $s$, and

$$
\begin{aligned}
& \operatorname{rank} K O^{0}(X)=\operatorname{rank} K O^{-4}(X)=t_{0}, \\
& \operatorname{rank} K O^{-2}(X)=\operatorname{rank} K O^{-6}(X)=t_{1} .
\end{aligned}
$$

By $(4-1)$ the extension of $\bigoplus_{p+q=2 i+1} E_{\infty}^{p, q}$ to $K O^{2 i+1}(X)$ is trivial. Thus if $X$ is a compact irreducible Hermitian symmetric space, we have

$$
\begin{aligned}
\operatorname{dim}_{z_{2}} K O^{2 i+1}(X) & =\operatorname{dim}_{z_{2}} \bigoplus_{p+q=2 i+1} E_{\infty}^{p, q} \\
& =\operatorname{dim}_{z_{2}} \underset{p=2 i+2(\bmod 8)}{\bigoplus} E_{\infty}^{p,-1} \\
& =\operatorname{dim}_{z_{2}} \bigoplus_{p=2 i+2(\bmod 8)} H^{p}\left(H^{*}(X) ; S q^{2}\right) \\
& =s_{i+1} .
\end{aligned}
$$

The proof of Corollary 2 is done.

Now we list the order of $K O^{*}(X)$, which is determined by $t_{\delta}$ and $s_{\varepsilon}$ as in Corollary 2.

We prepare a lemma for the cases $X=S p(n) / U(n)$ and $X=S O(2 n) /$ $U(n)$. Let $R_{n}^{*}$ be the exterior algebra over $\boldsymbol{Z}_{2}$ defined by

$$
R_{n}^{*}=\wedge\left(e_{1}, e_{2}, \cdots, e_{n}\right) \text {, with } \operatorname{deg} e_{i} \equiv 1(\bmod 4),
$$

and

$$
\rho(n, i)=\operatorname{dim} \underset{d \equiv i(\bmod 4)}{\bigoplus} R_{n}^{d}
$$


Of course, $\rho(n, i)$ equals to $\sum_{d \equiv i(\bmod 4)}\left(\begin{array}{l}n \\ d\end{array}\right)$. But to get more concrete description, we solve the next equations:

$$
\begin{aligned}
\rho(1,0) & =\rho(1,1)=1, \rho(1,2)=\rho(1,3)=0, \\
\rho(n+1, i) & =\rho(n, i-1)+\rho(n, i) .
\end{aligned}
$$

We have

PROPOSITION 4.1.

$$
\begin{aligned}
& \rho(n, 0)=2^{n-2}+\frac{\sqrt{-1}}{2}\left(\alpha^{n-2}-\beta^{n-2}\right), \\
& \rho(n, 1)=2^{n-2}+\frac{1}{2}\left(\alpha^{n-2}+\beta^{n-2}\right), \\
& \rho(n, 2)=2^{n-2}-\frac{\sqrt{-1}}{2}\left(\alpha^{n-2}-\beta^{n-2}\right), \\
& \rho(n, 3)=2^{n-2}-\frac{1}{2}\left(\alpha^{n-2}+\beta^{n-2}\right),
\end{aligned}
$$

where $\alpha=1+\sqrt{-1}, \beta=1-\sqrt{-1}$, thus we have

\begin{tabular}{|c|cccc|}
\hline$n$ & $\rho(n, 0)$ & $\rho(n, 1)$ & $\rho(n, 2)$ & $\rho(n, 3)$ \\
\hline $4 k$ & $2^{4 k-2}+(-1)^{k} 2^{2 k-1}$ & $2^{4 k-2}$ & $2^{4 k-2}-(-1)^{k} 2^{2 k-1}$ & $2^{4 k-2}$ \\
$4 k+1$ & $2^{4 k-1}+(-1)^{k} 2^{2 k-1}$ & $2^{4 k-1}+(-1)^{k} 2^{2 k-1}$ & $2^{4 k-1}-(-1)^{k} 2^{2 k-1}$ & $2^{4 k-1}-(-1)^{k} 2^{2 k-1}$ \\
$4 k+2$ & $2^{4 k}$ & $2^{4 k}+(-1)^{k} 2^{2 k}$ & $2^{4 k}$ & $2^{4 k}-(-1)^{k} 2^{2 k}$ \\
$4 k+3$ & $2^{4 k+1}-(-1)^{k} 2^{2 k}$ & $2^{4 k+1}+(-1)^{k} 2^{2 k}$ & $2^{4 k+1}+(-1)^{k} 2^{2 k}$ & $2^{4 k+1}-(-1)^{k} 2^{2 k}$ \\
\hline
\end{tabular}

From this, in the case $X=S p(n) / U(n)$, we get $s_{\varepsilon}$ 's by Theorem 2.1. $t_{\delta}$ 's are obtained by $(2-1)$.

THEOREM 4.2. For $X=S p(n) / U(n)$,

$$
\begin{aligned}
& t_{0}=t_{1}=2^{n-1}, \\
& s_{\varepsilon}=\rho\left(\left[\frac{n+1}{2}\right], \varepsilon\right) .
\end{aligned}
$$




\begin{tabular}{|c|cccc|}
\hline$n$ & $s_{0}$ & $s_{1}$ & $s_{2}$ & $s_{3}$ \\
\hline $8 k$ & $2^{4 k-2}+(-1)^{k} 2^{2 k-1}$ & $2^{4 k-2}$ & $2^{4 k-2}-(-1)^{k} 2^{2 k-1}$ & $2^{4 k-2}$ \\
$8 k+1$ & $2^{4 k-1}+(-1)^{k} 2^{2 k-1}$ & $2^{4 k-1}+(-1)^{k} 2^{2 k-1}$ & $2^{4 k-1}-(-1)^{k} 2^{2 k-1}$ & $2^{4 k-1}-(-1)^{k} 2^{2 k-1}$ \\
$8 k+2$ & $2^{4 k-1}+(-1)^{k} 2^{2 k-1}$ & $2^{4 k-1}+(-1)^{k} 2^{2 k-1}$ & $2^{4 k-1}-(-1)^{k} 2^{2 k-1}$ & $2^{4 k-1}-(-1)^{k} 2^{2 k-1}$ \\
$8 k+3$ & $2^{4 k}$ & $2^{4 k}+(-1)^{k} 2^{2 k}$ & $2^{4 k}$ & $2^{4 k}-(-1)^{k} 2^{2 k}$ \\
$8 k+4$ & $2^{4 k}$ & $2^{4 k}+(-1)^{k} 2^{2 k}$ & $2^{4 k}$ & $2^{4 k}-(-1)^{k} 2^{2 k}$ \\
$8 k+5$ & $2^{4 k+1}-(-1)^{k} 2^{2 k}$ & $2^{4 k+1}+(-1)^{k} 2^{2 k}$ & $2^{4 k+1}+(-1)^{k} 2^{2 k}$ & $2^{4 k+1}-(-1)^{k} 2^{2 k}$ \\
$8 k+6$ & $2^{4 k+1}-(-1)^{k} 2^{2 k}$ & $2^{4 k+1}+(-1)^{k} 2^{2 k}$ & $2^{4 k+1}+(-1)^{k} 2^{2 k}$ & $2^{4 k+1}-(-1)^{k} 2^{2 k}$ \\
$8 k+7$ & $2^{4 k+2}-(-1)^{k} 2^{2 k+1}$ & $2^{4 k+2}$ & $2^{4 k+2}+(-1)^{k} 2^{2 k+1}$ & $2^{4 k+2}$ \\
\hline
\end{tabular}

When $X=S O(2 n) / U(n)$, the next result is obtained by the similar computation as above from Theorem 2.3 and $(2-3)$.

THEOREM 4.3. For $X=S O(2 n) / U(n)$,

$$
\begin{aligned}
& t_{0}=t_{1}=2^{n-2}, \\
& s_{\varepsilon}= \begin{cases}\rho\left(\left[\frac{n}{2}\right], 1-\varepsilon\right), & \text { if } n \equiv 2(\bmod 4), \\
\rho\left(\left[\frac{n}{2}\right],-\varepsilon\right), & \text { otherwise. }\end{cases}
\end{aligned}
$$

\begin{tabular}{|c|cccc|}
\hline$n$ & $s_{0}$ & $s_{1}$ & $s_{2}$ & $s_{3}$ \\
\hline $8 k$ & $2^{4 k-2}+(-1)^{k} 2^{2 k-1}$ & $2^{4 k-2}$ & $2^{4 k-2}-(-1)^{k} 2^{2 k-1}$ & $2^{4 k-2}$ \\
$8 k+1$ & $2^{4 k-2}+(-1)^{k} 2^{2 k-1}$ & $2^{4 k-2}$ & $2^{4 k-2}-(-1)^{k} 2^{2 k-1}$ & $2^{4 k-2}$ \\
$8 k+2$ & $2^{4 k-1}+(-1)^{k} 2^{2 k-1}$ & $2^{4 k-1}+(-1)^{k} 2^{2 k-1}$ & $2^{4 k-1}-(-1)^{k} 2^{2 k-1}$ & $2^{4 k-1}-(-1)^{k} 2^{2 k-1}$ \\
$8 k+3$ & $2^{4 k-1}+(-1)^{k} 2^{k-1}$ & $2^{4 k-1}-(-1)^{k} 2^{2 k-1}$ & $2^{4 k-1}-(-1)^{k} 2^{2 k-1}$ & $2^{4 k-1}+(-1)^{k} 2^{2 k-1}$ \\
$8 k+4$ & $2^{4 k}$ & $2^{4 k}-(-1)^{k} 2^{2 k}$ & $2^{4 k}$ & $2^{4 k}+(-1)^{k} 2^{2 k}$ \\
$8 k+5$ & $2^{4 k}$ & $2^{4 k}-(-1)^{k} 2^{2 k}$ & $2^{4 k}$ & $2^{4 k}+(-1)^{k} 2^{2 k}$ \\
$8 k+6$ & $2^{4 k+1}+(-1)^{k} 2^{2 k}$ & $2^{4 k+1}-(-1)^{k} 2^{2 k}$ & $2^{4 k+1}-(-1)^{k} 2^{2 k}$ & $2^{4 k+1}+(-1)^{k} 2^{2 k}$ \\
$8 k+7$ & $2^{4 k+1}-(-1)^{k} 2^{2 k}$ & $2^{4 k+1}-(-1)^{k} 2^{2 k}$ & $2^{4 k+1}+(-1)^{k} 2^{2 k}$ & $2^{4 k+1}+(-1)^{k} 2^{2 k}$ \\
\hline
\end{tabular}

When $X=Q_{n}$, by $(2-5)$ and Theorem 2.5, we have

THEOREM 4.4. For $X=Q_{n}$, 


\begin{tabular}{|c|cccccc|}
\hline$n$ & $t_{0}$ & $t_{1}$ & $s_{0}$ & $s_{1}$ & $s_{2}$ & $s_{3}$ \\
\hline $8 k$ & $4 k+2$ & $4 k$ & 2 & 0 & 0 & 0 \\
$8 k+1$ & $4 k+1$ & $4 k+1$ & 1 & 1 & 0 & 0 \\
$8 k+2$ & $4 k+2$ & $4 k+2$ & 1 & 2 & 1 & 0 \\
$8 k+3$ & $4 k+2$ & $4 k+2$ & 1 & 1 & 0 & 0 \\
$8 k+4$ & $4 k+4$ & $4 k+2$ & 2 & 0 & 0 & 0 \\
$8 k+5$ & $4 k+3$ & $4 k+3$ & 1 & 0 & 0 & 1 \\
$8 k+6$ & $4 k+4$ & $4 k+4$ & 1 & 0 & 1 & 2 \\
$8 k+7$ & $4 k+4$ & $4 k+4$ & 1 & 0 & 0 & 1 \\
\hline
\end{tabular}

For $X=E I I I$, by $(3-1)$ and Theorem 3.1 and for $X=E V I I$, by $(3-3)$ and Theorem 3.6, we have the following table.

THEOREM 4.5. For the exceptional types,

\begin{tabular}{|c|cccccc|}
\hline$X$ & $t_{0}$ & $t_{1}$ & $s_{0}$ & $s_{1}$ & $s_{2}$ & $s_{3}$ \\
\hline$E I I I$ & 15 & 12 & 3 & 0 & 0 & 0 \\
\hline$E V I I$ & 28 & 28 & 1 & 3 & 3 & 1 \\
\hline
\end{tabular}

\section{References}

[1] BOTT, R., The space of loops on a Lie group, Michigan Math. J. 5 (1958), 35-61.

[2] BOREL, A. and HIRZEBRUCH, F., Characteristic classes and homogeneous spaces, Amer. J. Math. 80 (1958), 458-538.

[ 3 ] FUJII, M., KO-groups of projective spaces, OSAKA J. Math. 4 (1967), 141-49.

[4] Hogger, S. G., On KO theory of Grassmannians, Quart. J. Math. Oxford (2) 20 (1969), 447-63.

[5] IShitoyA, K., Squaring Operations in the Hermitian Symmetric Spaces, To Appear.

[6] KONO, A. - HARA, S., KO-theory of Complex Grassmannian Manifold, J. Math. Kyoto Univ., to appear.

[7] Kono, A.-Kozima. K., The Mod 2 Homology of the Space of Loops on the Exceptional Lie Groups, Proc. Royal Soc. Edinburgh 112A (1989), 187-202.

[8] Milnor, J.W. and MOORE J.C., On the Structure of Hopf Algebras, Ann. Math. 81 (1965), 211-264.

[9] Toda, H. and Watanabe, T., The Integral Cohomology Ring of $F_{4} / T$ and $E_{6} / T, \mathrm{~J}$. Math. Kyoto Univ. 14-2 (1974), 257-286.

[10] Watanabe, T., The Integral Cohomology Ring of the symmetric space EVII, J. Math. Kyoto Univ. 15-2 (1975), 363-385.

Department of Mathematics Kyoto University Sakyo-ku, Kyoto 606 Japan 